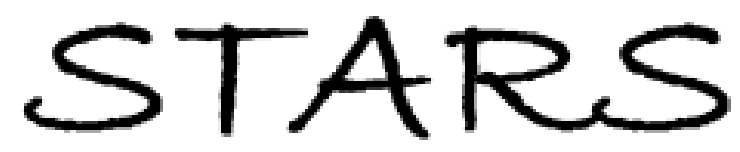

University of Central Florida

STARS

$1-1-2009$

\title{
Optical and electron beam studies of carrier transport in quasibulk GaN
}

\author{
Y. Lin \\ University of Central Florida \\ E. Flitsyian \\ University of Central Florida \\ L. Chernyak \\ University of Central Florida \\ T. Malinauskas \\ R. Aleksiejunas
}

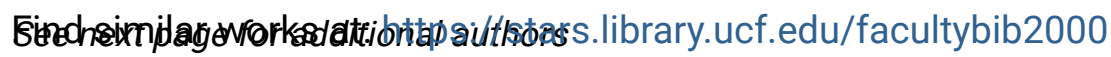

University of Central Florida Libraries http://library.ucf.edu

This Article is brought to you for free and open access by the Faculty Bibliography at STARS. It has been accepted for inclusion in Faculty Bibliography 2000s by an authorized administrator of STARS. For more information, please contactSTARS@ucf.edu.

\section{Recommended Citation}

Lin, Y.; Flitsyian, E.; Chernyak, L.; Malinauskas, T.; Aleksiejunas, R.; Jarasiunas, K.; Lim, W.; Pearton, S. J.; and Gartsman, K., "Optical and electron beam studies of carrier transport in quasibulk GaN" (2009). Faculty Bibliography 2000s. 1804.

https://stars.library.ucf.edu/facultybib2000/1804

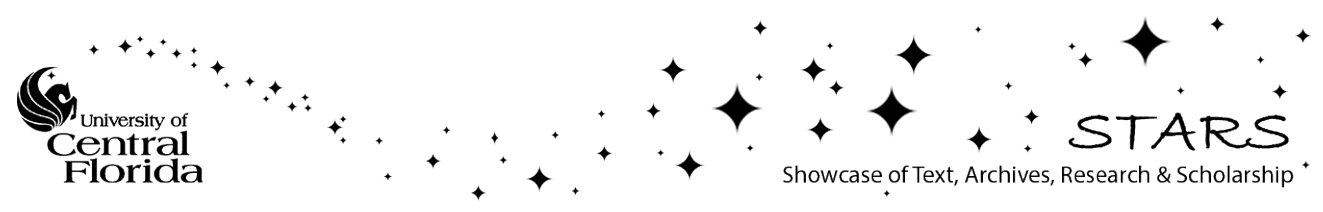




\section{Authors}

Y. Lin, E. Flitsyian, L. Chernyak, T. Malinauskas, R. Aleksiejunas, K. Jarasiunas, W. Lim, S. J. Pearton, and K. Gartsman 


\section{Optical and electron beam studies of carrier transport in quasibulk GaN}

Cite as: Appl. Phys. Lett. 95, 092101 (2009); https://doi.org/10.1063/1.3220062

Submitted: 07 August 2009 . Accepted: 14 August 2009 . Published Online: 31 August 2009

Y. Lin, E. Flitsyian, L. Chernyak, T. Malinauskas, R. Aleksiejunas, K. Jarasiunas, W. Lim, S. J. Pearton, and K. Gartsman

\section{ARTICLES YOU MAY BE INTERESTED IN}

Minority carrier diffusion length in GaN: Dislocation density and doping concentration dependence

Applied Physics Letters 86, 052105 (2005); https://doi.org/10.1063/1.1861116

Carrier dynamics in bulk GaN

Journal of Applied Physics 111, 023702 (2012); https://doi.org/10.1063/1.3673851

Nonradiative recombination at threading dislocations in n-type GaN: Studied by cathodoluminescence and defect selective etching

Applied Physics Letters 92, 231909 (2008); https://doi.org/10.1063/1.2928226

\section{Applied Physics Letters}

Mid-IR and THz frequency combs special collection

Read Now! 


\title{
Optical and electron beam studies of carrier transport in quasibulk GaN
}

\author{
Y. Lin, ${ }^{1}$ E. Flitsyian, ${ }^{1}$ L. Chernyak, ${ }^{1, a)}$ T. Malinauskas, ${ }^{2}$ R. Aleksiejunas, ${ }^{2}$ K. Jarasiunas, ${ }^{2}$ \\ W. Lim, ${ }^{3}$ S. J. Pearton, ${ }^{3}$ and K. Gartsman ${ }^{4}$ \\ ${ }^{1}$ Department of Physics, University of Central Florida, Orlando, Florida 32816-2385, USA \\ ${ }^{2}$ Institute of Applied Research, Vilnius University, 10222 Vilnius, Lithuania \\ ${ }^{3}$ Department of Materials Science and Engineering, University of Florida, Gainesville, \\ Florida 32611-6400, USA \\ ${ }^{4}$ Weizmann Institute of Science, Rehovot 76100, Israel
}

(Received 7 August 2009; accepted 14 August 2009; published online 31 August 2009)

\begin{abstract}
Variable temperature light-induced transient grating technique combined with electron beam-induced current measurements in situ in a scanning electron microscope were employed for carrier transport studies in quasibulk hydride-vapor phase epitaxy grown undoped GaN layers. Diffusion length of carriers independently determined from both techniques was found to increase with temperature in the range from 70 to $400 \mathrm{~K}$. This increase was attributed to the temperature-induced growth of carrier lifetime, as was confirmed by light-induced transient grating measurements below $300 \mathrm{~K}$ and by cathodoluminescence above room temperature. (C) 2009 American Institute of Physics. [DOI: 10.1063/1.3220062]
\end{abstract}

The electronic and photonic device applications which became possible with the maturing of GaN technology ${ }^{1}$ also require reliable testing techniques allowing determination of fundamental materials properties, such as carrier lifetime, diffusion coefficient, and diffusion length. light-induced transient grating (LITG) is one of these techniques, which allows independent determination of ambipolar diffusion coefficient, $D_{a}$, and lifetime, $\tau_{R}$, of nonequilibrium carriers in one experiment. ${ }^{2}$ Ambipolar carrier diffusion length, $L_{a}$, can be, therefore, calculated as $L_{a}=\sqrt{D_{a} \tau_{R}}$. Another technique is electron beam-induced current (EBIC), ${ }^{3}$ which in contrast to LITG allows direct measurements of minority carrier diffusion length, $L$, from a simple experiment. These two techniques are, therefore, complementary in a sense that, while in LITG, the values of $D_{a}$ and $\tau_{R}$ are simultaneously extracted from the experimental data, in the EBIC technique, the diffusion length value is directly measured. In this letter we report minority carrier transport measurements in hydridevapor phase epitaxy (HVPE) grown quasibulk GaN using both techniques and complement them with cathodoluminescence (CL) spectroscopy in situ in scanning electron microscope (SEM).

In the LITG technique, ${ }^{2}$ a sample is excited by an interference pattern $I(\mathrm{x})=I_{0}[1+\cos (2 \pi \mathrm{x} / \Lambda)]$ of two coherent laser pulses; here $I_{0}$ stands for excitation fluence and $\Lambda$ denotes a period of the interference pattern. Nonequilibrium carriers are photoexcited in the regions of constructive interference. These carriers give rise to a periodical modulation of the refractive index $\Delta n$ in the material, or transient diffraction grating. In the given case, $\Delta n$ is proportional to the concentration of photoexcited carriers. In contrast to spatially homogeneous excitation, LITG decays due to two physical mechanisms: nonequilibrium carrier recombination and diffusion along the grating vector. Therefore, the LITG technique allows simultaneous determination of two characteristic quantities: carrier lifetime, $\tau_{R}$, and ambipolar diffusion coefficient, $D_{a}$, which in turn give the values for diffu-

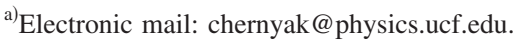

sion length according to the above-referenced equation.

Temporal evolution of instantaneous carrier modulation, $\Delta N$, is experimentally monitored by diffraction of a delayed probe beam. In the case of exponential decay of the diffraction efficiency, $\eta$, the grating decay time, $\tau_{G}$, can be introduced as $\eta(t) \propto \exp \left(-2 t / \tau_{G}\right)$ where $\tau_{G}$ is equal to

$$
1 / \tau_{G}=1 / \tau_{R}+1 / \tau_{D}=1 / \tau_{R}+4 \pi^{2} D_{a} / \Lambda^{2},
$$

here $\tau_{D}$ and $D_{a}$ stand for diffusive grating decay time and ambipolar diffusion coefficient, respectively. We note that $\tau_{D}$ in the experiment is controlled by choosing $\Lambda$. As it is evident from Eq. (1), $\tau_{R}$, and $D_{a}$ can be obtained from the $1 / \tau_{G}=\mathrm{f}\left(1 / \Lambda^{2}\right)$ plot.

A direct method for the extraction of minority carrier diffusion length, $L$, from the EBIC line-scan is described in Ref. 3. If the incident electron beam is perpendicular to a planar Schottky barrier, created on the material's top surface, the EBIC signal, $I$, decays with the beam-to-barrier distance, $d$, according to the relationship

$$
I=\mathrm{A} d^{\alpha} \exp (-d / L)
$$

here $A$ is a constant, and $\alpha$ is a coefficient that depends on the surface recombination velocity, $v_{s}$. This coefficient varies from $\alpha=-1 / 2$, for $v_{s}=0$, to $\alpha=-3 / 2$, for infinite $v_{s}$. Since infinite surface recombination velocity is very unlikely in view of the excellent luminescence efficiency of $\mathrm{GaN}$, the value of $\alpha=-1 / 2$ was used in this work. Equation (2) yields a straight line relationship for a plot of $\ln \left(I \times d^{1 / 2}\right)$ versus $d$. The diffusion length, $L$, can be then obtained directly as $-1 /$ slope.

For present LITG studies, (YAG:Nd) laser pulses of 23 ps duration and $355 \mathrm{~nm}$ wavelength were used to record a diffraction grating. The incident fluence was varied within $0.1-5 \mathrm{~mJ} / \mathrm{cm}^{2}$. The band-to-band absorption of the recording light resulted in small absorption depth $(\sim 150 \mathrm{~nm})$ and, thus, high excess carrier concentration $\left(\sim 5 \times 10^{18} \mathrm{~cm}^{-3}\right.$ at the end of the pulse). Carrier diffusion into the bulk rapidly diluted the generated carrier plasma density and increased 


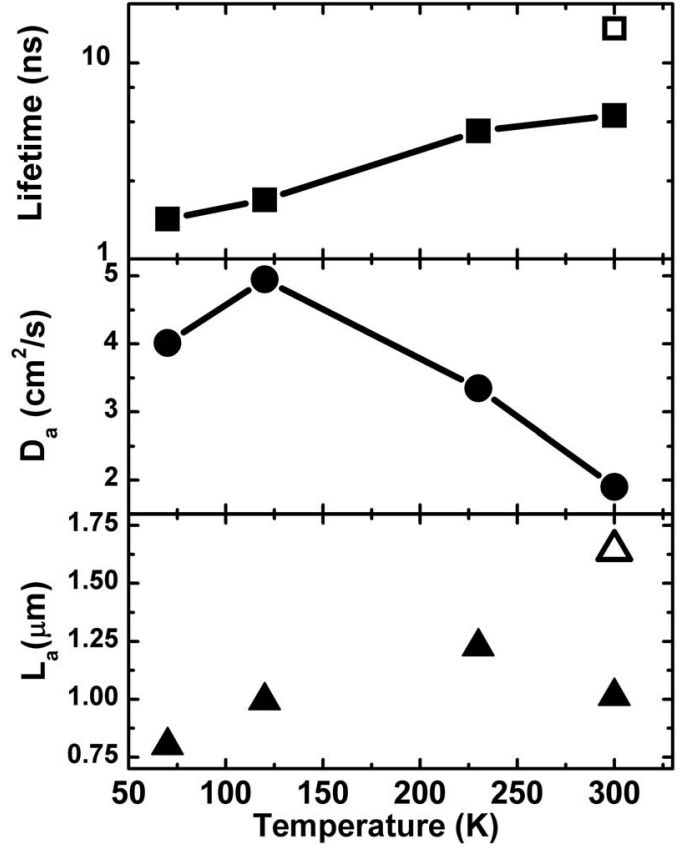

FIG. 1. Temperature dependence of carrier lifetime, ambipolar diffusion coefficient, $D_{a}$, and ambipolar diffusion length, $L_{a}$, measured in HVPE GaN quasibulk epitaxial layer by LITG technique. The full (open) points correspond to high (low) excitation level of $\sim 5 \times 10^{18} \mathrm{~cm}^{-3}\left(\sim 10^{16} \mathrm{~cm}^{-3}\right)$.

the excited layer thickness up to $1-2 \mu \mathrm{m}$. ${ }^{4}$ The temperature in LITG experiments was varied using a closed-cycle He-gas cryostat under a pressure of $\sim 10^{-7}$ Torr.

EBIC experiments were carried out in situ in Philips XL-30 TMP SEM under a $30 \mathrm{kV}$ electron beam accelerating voltage, which corresponds to several micron beam penetration depth in the material and nonequilibrium electron-hole pair density of $\sim 1 \times 10^{19} \mathrm{~cm}^{-3}$. 5 EBIC signal line-scans were recorded using homemade software, a Stanford research system low-noise current amplifier and a Keithley 2000 digital multimeter, employed as a digitizer. The sample temperature was varied in situ from 25 to $125^{\circ} \mathrm{C}$ using a specially designed hot stage and an external temperature controller (Gatan). At each temperature, the EBIC measurements were conducted at 5-7 different locations and the mean values for minority carrier diffusion length were calculated. A series of variable temperature CL measurements in situ in SEM (Ref. 6) was carried out in the same locations as the EBIC measurements using an integrated Gatan MonoCL3 system.

Free-standing $400 \mu \mathrm{m}$ thick, crack-free $\mathrm{GaN}$ layers were grown by HVPE on a two-step epitaxial lateral overgrown GaN template on sapphire. ${ }^{7}$ The "as-grown" epitaxial layer was of $n$-type electrical conductivity with room temperature majority electron concentration of $\sim 1 \times 10^{17} \mathrm{~cm}^{-3}$ and mobility of $\sim 750 \mathrm{~cm}^{2} / \mathrm{V}$ s. Polychromatic CL mapping revealed a threading dislocation density of $10^{6} \mathrm{~cm}^{-2}$. Schottky barriers, needed for EBIC measurements, were fabricated by electron beam evaporation of $80 \mathrm{~nm}$ thick $\mathrm{Ni} / \mathrm{Au}$ layers and subsequent lift-off.

The measurements of LITG kinetics at several grating periods allowed to separate diffusion and recombination processes, and to measure diffusion coefficient and carrier lifetime. Figure 1 shows carrier lifetime, ambipolar diffusion coefficient, and ambipolar diffusion length values obtained using Eq. (1) in the HVPE epitaxial layer at low tempera-

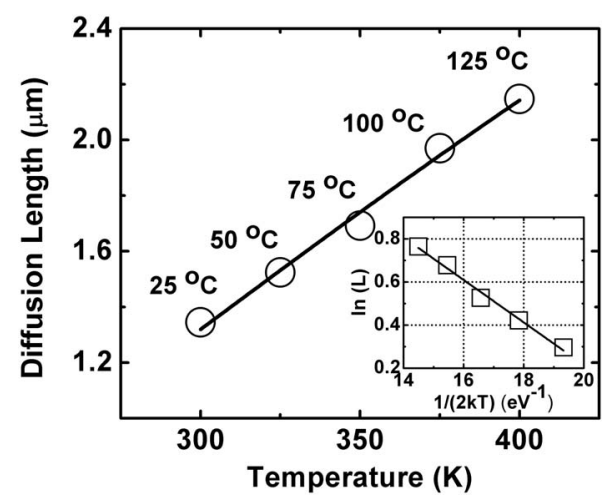

FIG. 2. Temperature dependence for $L$ directly obtained from the EBIC measurements on HVPE quasibulk epitaxial layer and the fit using Eq. (3) (solid curve). Inset: Arrhenius plot of the same data yielding the activation energy of $99 \pm 3 \mathrm{meV}$.

tures. While the carrier lifetime shows a significant growth with increasing temperature, the ambipolar diffusion coefficient exhibits decay in this temperature range. The ambipolar diffusion length, being a product of these two quantities, exhibits a modest growth, with peak value $L_{a}=1.25 \mu \mathrm{m}$ at $230 \mathrm{~K}$ under the given high-excitation conditions. We note that carrier lifetime under high-excitation is governed by fast radiative recombination. Measurements under few orders of magnitude lower excess carrier density, exhibited the dominance of the nonradiative recombination with the lifetime of $15 \mathrm{~ns}$ in the given crystal. ${ }^{8}$ Correspondingly, the ambipolar diffusion length value increased up to $1.7 \mu \mathrm{m}$ at excess carrier density of $\sim 10^{16} \mathrm{~cm}^{-3}$ (open point in Fig. 1). The ambipolar diffusion length $L_{a}=1.7 \mu \mathrm{m}$ at $300 \mathrm{~K}$ corresponds to the minority hole diffusion length $L=1.2 \mu \mathrm{m}$, as $D_{a} \approx 2 D_{h}$.

The temperature dependence for $L$, directly extracted from EBIC measurements in 300-400 K range, shown in the Fig. 2, was fitted using the following equation:

$$
L=L_{0} \exp \left(-\frac{\Delta E_{A, T}}{2 k T}\right),
$$

where $L_{0}$ is a scaling factor, $k$ is the Boltzmann constant, $\Delta E_{A, T}$ is the thermal activation energy, and $T$ is the absolute temperature. The inset of Fig. 2 shows the Arrhenius plot for $L(T)$, from which the value of $\Delta E_{A, T}$ of about $100 \mathrm{meV}$ was obtained as the slope. It is also important to note that $L$ results, obtained by LITG and EBIC techniques, are in reasonable agreement: 1.2 versus $1.3 \mu \mathrm{m}$ at room temperature, respectively.

The role of increasing carrier lifetime is supported by the results of CL measurements, presented in the inset of Fig. 3, which show CL spectra in 300-400 K range in the vicinity of the band-to-band transition. The maxima of these spectra exhibit a redshift with increasing temperature consistent with the increasing $\mathrm{GaN}$ band gap. $\mathrm{It}$ is also observed that the peak intensity, $I_{\mathrm{CL}}$, of the band-to-band luminescence decreases systematically with increasing temperature (cf. Fig. 3 ), providing direct evidence that the number of recombination events decreases $\left(I_{\mathrm{CL}} \propto B \times n \times p\right.$, where $B$ is a bimolecular recombination coefficient; $n$ and $p$ are the nonequilibrium densities of electrons and holes, respectively). The $I_{\mathrm{CL}}$ signal decreases exponentially according to the equation below: ${ }^{10}$ 


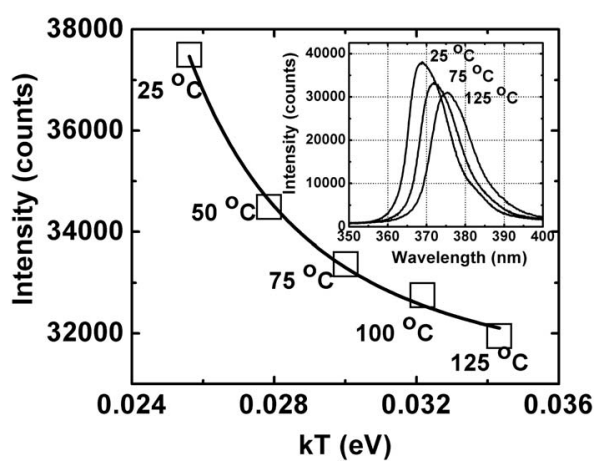

FIG. 3. Temperature dependence for the peak CL intensity in HVPE quasibulk epitaxial layer and the fit using Eq. (4). The fit resulted in the activation energy of $112 \pm 5 \mathrm{meV}$. Inset: CL spectra taken at different temperatures in the vicinity of the band-to-band transition. The redshift in CL maxima, observed with increasing temperature, is due to the temperature-induced widening of the $\mathrm{GaN}$ band gap.

$$
I_{\mathrm{CL}}=\frac{A}{\left[1+C \exp \left(-\frac{\Delta E_{A}}{k T}\right)\right]},
$$

where $A$ and $C$ are the scaling factors, and $\Delta E_{A}$ is the activation energy, similar in nature to that in Eq. (3). Based on the fit shown in Fig. 3, the activation energy was determined to be about $115 \mathrm{meV}$. This energy is in reasonable agreement with that obtained in the same temperature range from variable temperature EBIC measurements, which suggest that the same underlying process is responsible for both the increase in the diffusion length and the CL intensity decay. This process is outlined below.

Nonequilibrium electron-hole pairs, generated by the SEM (or laser) beam, subsequently annihilate by recombining with each other via nonradiative and radiative (bimolecular) recombination. It has been shown that for high nonequilibrium carrier concentration and low temperature (10-300 $\mathrm{K})$ the bimolecular recombination is a dominant channel in high quality HVPE-grown samples. ${ }^{11}$ Since the bimolecular recombination coefficient, $B$, is proportional to $T^{-3 / 2},{ }^{12}$ the radiative carrier lifetime becomes longer at higher temperatures. Above room temperature the CL signal decay also shows the decreasing probability of electron-hole recombination. This means that nonequilibrium electron-hole pairs exist for longer periods of time and, as a result, diffuse longer distances before undergoing recombination, thus increasing
$L$ values. As it was demonstrated in LITG experiments (cf. Fig. 1), the impact of diffusivity on $L_{a}$ versus $T$ dependence is opposite. The diffusivity decreases with temperature, most likely, due to optical phonon scattering-limited carrier mobility.

In conclusion, LITG, EBIC, and CL measurements were carried out on quasibulk HVPE GaN material. Diffusion length independently determined from LITG and EBIC techniques was found to increase with temperature in the range from $\sim 70$ to $400 \mathrm{~K}$. This increase was attributed to the temperature-induced growth of radiative carrier lifetime as was independently confirmed by LITG and CL. Finally, the $L$ values at $300 \mathrm{~K}$, obtained by LITG and EBIC techniques, are in good agreement, which indicates authenticity of the above-reported results.

Research at UCF was supported in part by NSF Grant No. ECCS 0900971 and NATO Grant No. SfP 981939. The work at UF was supported by NSF Grant No. DMR 0703340. VU acknowledges the support by NATO Grant No.SfP 981939 and Lithuanian Grant No. SSSF V-02/2009. Research at Weizmann Institute was conducted under NATO Grant No.SfP 981939.

${ }^{1}$ T. Anderson, F. Ren, S. Pearton, B. S. Kang, H. T. Wang, C. Y. Chang, and J. S. Lin, Sensors 9, 4669 (2009); M. Razeghi and R. McClintock, J. Cryst. Growth 311, 3067 (2009).

${ }^{2}$ H. J. Eichler, P. Gunter, and D. W. Pohl, Laser-Induced Dynamic Gratings (Springer, Berlin, 1986).

${ }^{3}$ D. S. H. Chan, V. K. S. Ong, and J. C. H. Phang, IEEE Trans. Electron Devices 42, 963 (1995).

${ }^{4}$ T. Malinauskas, K. Jarasiunas, S. Miasojedovas, S. Jursenas, B. Beaumont, and P. Gibart, Appl. Phys. Lett. 88, 202109 (2006).

${ }^{5}$ H. J. Leamy, J. Appl. Phys. 53, R51 (1982).

${ }^{6}$ O. Lopatiuk-Tirpak, L. Chernyak, Y. L. Wang, F. Ren, S. J. Pearton, K. Gartsman, and Y. Feldman, Appl. Phys. Lett. 90, 172111 (2007).

${ }^{7}$ D. Gogova, A. Kasic, H. Larsson, C. Hemmingsson, B. Monemar, F. Tuomisto, K. Saarinen, L. Dobos, B. Pecz, P. Gibart, and B. Beaumont, J. Appl. Phys. 96, 799 (2004).

${ }^{8}$ K. Jarasiunas, T. Malinauskas, R. Aleksiejunas, B. Monemar, V. Ralchenko, A. Gontar, and E. Ivakin, Mater. Sci. Forum 600, 1301 (2009).

${ }^{9}$ V. Bougrov, M. E. Levinshtein, S. L. Rumyantsev, and A. Zubrilov, in Properties of Advanced SemiconductorMaterials GaN, AlN, InN, BN, SiC, SiGe, edited by M. E. Levinshtein, S. L. Rumyantsev, and M. S. Shur, (Wiley, New York, 2001), pp. 1-30.

${ }^{10}$ D. S. Jiang, H. Jung, and K. Ploog, J. Appl. Phys. 64, 1371 (1988).

${ }^{11}$ T. Malinauskas, K. Jarašiūnas, R. Aleksiejūnas, D. Gogova, B. Monemar, B. Beaumont, and P. Gibart, Phys. Status Solidi B 243, 1426 (2006).

${ }^{12}$ A. Dmitriev and A. Oruzheinikov, J. Appl. Phys. 86, 3241 (1999). 